\title{
AN INVENTORY SYSTEM WITH BOTH DELAYED AND IMMEDIATE DELIVERY
}

\author{
PETER W. HOVEY, ${ }^{*}$ University of Dayton Research Institute \\ PETER PURDUE, ${ }^{* *}$ University of Kentucky
}

\begin{abstract}
An $M / G / \infty$ queue that is cleared whenever the level $Q$ is exceeded plays the role of the outstanding orders process in an inventory system. The stationary version of the process is examined and an interesting property of the variance is illustrated.
\end{abstract}

INFINITE-SERVER QUEUE

\section{Introduction}

Let us consider an inventory system in which single demands occur according to a Poisson process at rate $\lambda$, and each demand must be met at once. When a demand arrives, an item is removed from inventory and an order for a replacement is sent which requires a random length of time with d.f. $G$, for delivery. All outstanding orders are delivered as soon as a demand arrives to an empty system. Let $Q$ be the initial size of the inventory; then as soon as the system is completely depleted the number of outstanding items is $Q+1$. We shall assume here that $Q$ is known and examine the behavior of the inventory system.

Let $I(t)$ denote the number of items in stock, and $Z(t)$ the number of items on order at time $t$. Then for all $t, 0 \leqq Z(t) \leqq Q$, and

$$
I(t)=Q-Z(t) .
$$

The process $\{Z(t) ; t \geqq 0\}$ behaves as an $M / G / \infty$ queue that is cleared back to 0 at the time of entry into state $Q+1$. Following Miller (1972), it is easy to see that this process is regenerative and is built up from a tour $[X(t), T]$ where $X(t)$ is the content at $t$ of an $M / G / \infty$ queue and $T$ is the first-passage time of this process into state $Q+1$. Let $\left\{Z^{*}(t) ; t \geqq 0\right\}$ be the stationary version of the $Z$ process; we study the behavior of the variance of $Z^{*}(0)$ and, consequently, of the stationary inventory process. Related work on cleared processes is due to Stidham (1974) and Whitt (1981).

\footnotetext{
Received 25 May 1983.

* Postal address: 564 B Kettering Labs., University of Dayton Research Institute, 300 College Park Drive, Dayton, OH 45469, U.S.A.

** Postal address: Department of Statistics, University of Kentucky, Lexington, KY 40506, U.S.A.

Research supported in part by NSF Grant No. MCS-8102215-01.
} 


\section{Main results}

Let $T(k, Q)$ denote the time spent, during a tour, in state $k$. Then, from Ross (1970) we have

$$
P\left[Z^{*}(t)=k\right]=\frac{E[T(k, Q)]}{E(T)}, \quad k=0,1, \ldots, Q .
$$

Letting $q(t)=\lambda \int_{0}^{t}[1-G(x)] d x$, we have

$$
P\left[Z^{*}(t)=k\right]=\frac{1}{E(T)} \int_{0}^{\infty}\left\{\frac{e^{-q(t)} q^{k}(t)}{k !}-P[X(t)=k, T \leqq t]\right\} d t \quad(k \geqq 0) .
$$

This follows from the law of total probabilities. When $G$ is exponential with mean $1 / \mu$ a major simplification of (3) is possible.

Theorem 1. When the delivery time is exponential then
(i) $E(T)=\frac{1}{\lambda} \sum_{i=0}^{O}\left(\begin{array}{l}Q+1 \\ k+1\end{array}\right) \frac{k !}{\rho^{k}}$,
(ii) $E\left[Z^{*}(t)\right]=\rho\left[1-\frac{Q+1}{E(T)}\right]$
(iii) $\operatorname{Var}\left[Z^{*}(t)\right]=\rho\left[1-\frac{Q+1}{\lambda E(T)}\left\{\frac{Q+2}{2}-\rho\left(1-\frac{Q+1}{E(T)}\right)\right\}\right]$

where, $\rho=\lambda / \mu$.

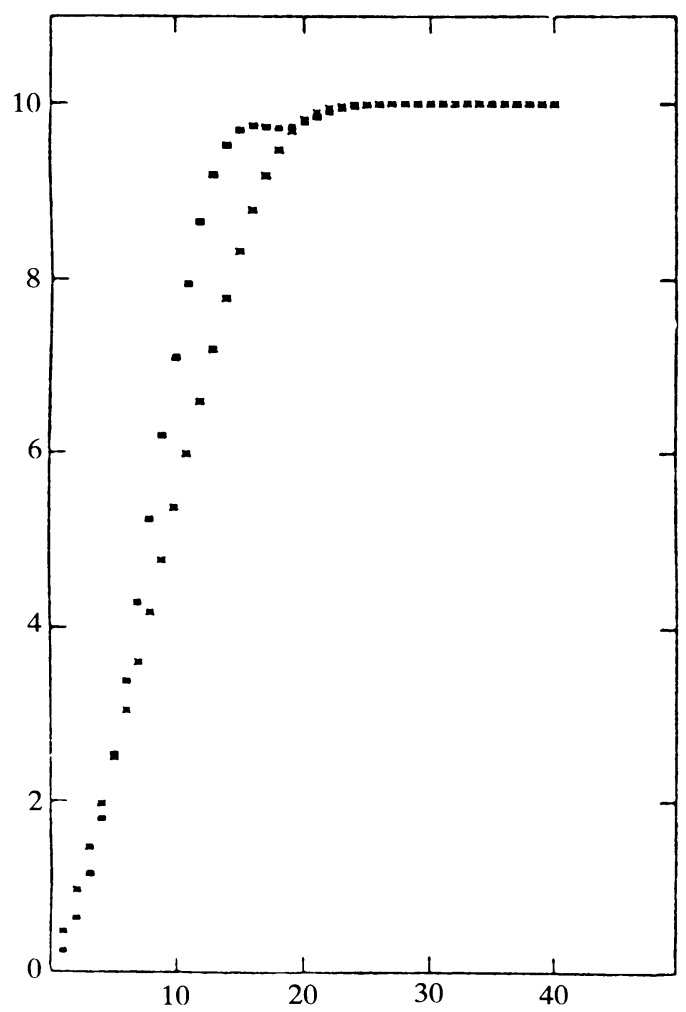

Figure 1. Mean and variance, $\rho=10$ 
Proof. The result (i) is well known. Result (ii) follows after some tedious algebra from Equation (3). An important step in deriving (ii) is to note that $P[X(t)=k, T \leqq t]=$ $\int_{0}^{t} P[X(t-s)=k \mid X(0)=Q+1] d s$. Result (iii) also follows directly. Now, from Equation $(1)$,

$$
E\left[I^{*}(t)\right]=Q-E\left[Z^{*}(t)\right], \quad \operatorname{Var}\left[I^{*}(t)\right]=\operatorname{Var}\left[Z^{*}(t)\right]
$$

\section{Comments}

As $Q$ increases, we expect that the mean and variance of $Z^{*}(t)$ should converge to $\rho$; a little algebra shows that this is indeed so. For the mean, $E\left[Z^{*}(t)\right]$ converges monotonically to $\rho$ as $Q \rightarrow \infty$. However, this is not so for $\operatorname{Var}\left[Z^{*}(t)\right]$. For $\rho$ small, the convergence is monotonic but it fails to be so as $\rho$ increases. We can best illustrate this by examining some numerical results. For $\rho=20$ the lack of monotonicity is obvious and this departure from monotone convergence increases as $\rho$ increases (see Figures 1 , 2 and 3; means are represented by crosses and variances by squares for increasing values of $Q$ ). Although we do not have an analytic result, the peak value of the variance seems to occur when $Q$ and $\rho$ are roughly equal. We can offer an informal explanation for the phenomenon. For a given value of $\rho$ when $Q$ is small there is very little chance that any orders will be delivered before the inventory level hits 0 and so there is little variability. When $Q$ is very large the system is for all intents and purposes an $M / M / \infty$ queue and this again is not very variable. The only possiblity for any high degree of variability is

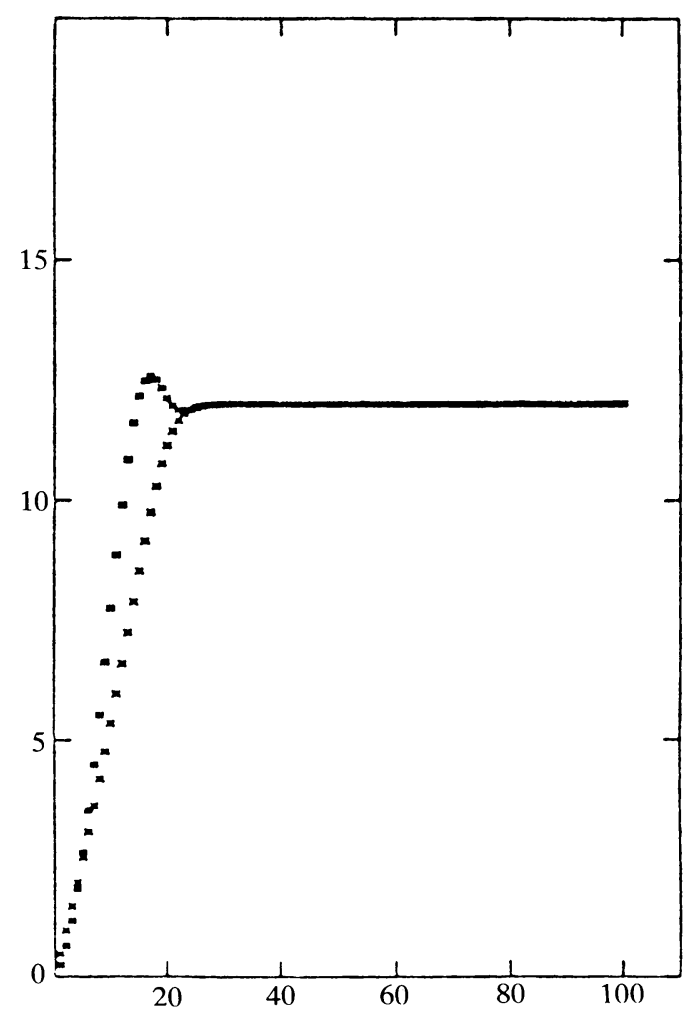

Figure 2. Mean and variance, $\rho=12$ 


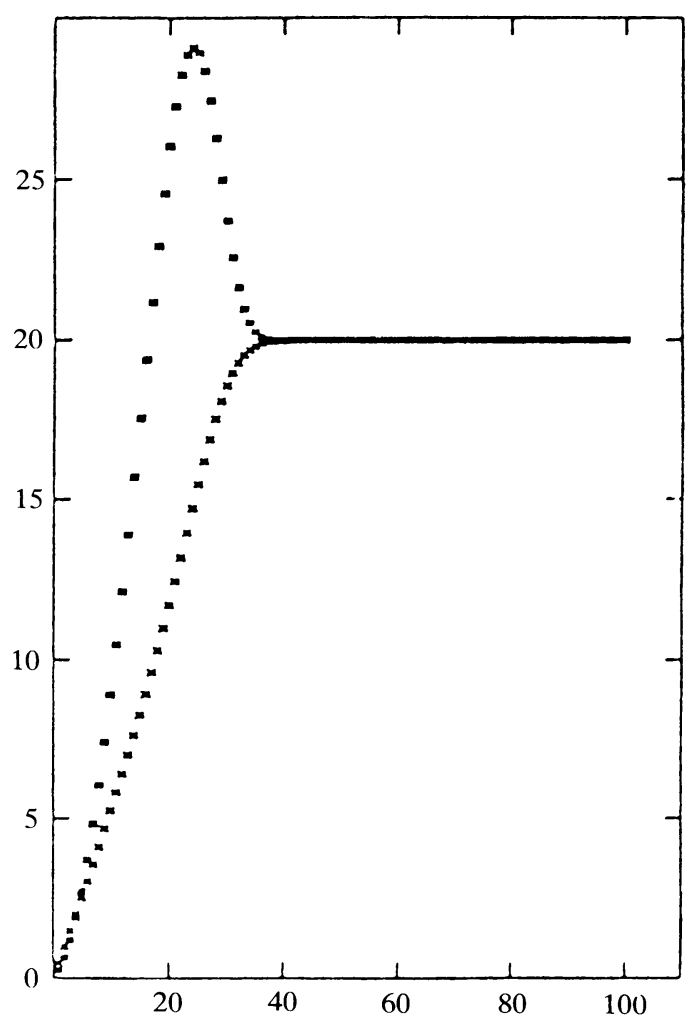

Figure 3. Mean and variance, $\rho=20$

when $Q$ is neither too big nor too small. We note that when $\rho$ is large and the queue size is small, the number of departures is small compared to the number of arrivals. In this case the system acts much like a pure birth or Poisson process. Stidham has shown that the limiting distribution for a Poisson process cleared at level $Q+1$ is uniform on $[0, Q]$. The mean and variance would then be $Q / 2$ and $[Q(Q+2)] / 12$, respectively. For large values of $\rho$, the curves of the variance of $V^{*}(0)$ tend to follow the variance of a uniform distribution. As $Q$ gets larger, and into the 'likely' range of the limiting distribution of an $M / M / \infty$ queue, the variance decreases again.

\section{References}

Miller, D. R. (1972) Existence of limits in regenerative processes. Ann. Math. Statist. 43, $1275-1282$.

Ross, S. M. (1970) Applied Probability Models with Optimization Applications. Holden-Day, San Francisco.

Stidham, S. (1974) Stochastic clearing systems. Stoch. Proc. Appl. 4, 85-113.

WHITT, W. (1981) The stationary distribution of a stationary clearing process. Operat. Res. 29, 294-308. 